\title{
Photopolymerizable monomer formulations for nanoporous proton conducting membranes
}

\author{
M. Kellner ${ }^{a}$, P. Radovanovic ${ }^{a, b}$, J. Matovic $^{b}$, R. Liska ${ }^{a}$ \\ a Institute of Applied Synthetic Chemistry, TU Wien, Getreidemarkt 9/163MC; 1060 Vienna, Austria \\ ${ }^{\mathrm{b}}$ Institute of Sensor and Actuator Systems, TU Wien, Floragasse 7; 1040 Vienna, Austria
}

\begin{abstract}
Several monomers and crosslinker in a broad range of concentrations in water and 1-Methyl-2-pyrrolidone (NMP) respectively were screened for their mechanical properties, water uptake and conductivity by photo polymerization with a polar photo initiator in porous membranes made of polypropylene and polyethersulfone respectively. As conductive polymer, primarily poly(2-acrylamido-2-methylpropane sulfonic acid) (PAMPS) and poly(2-sulfoethyl methacrylate) (PSEM) respectively as well as polymers of phosphonic acid containing monomers or newly synthesized monomers were used. The conductive monomers were crosslinked with varying hydrophobic and hydrophilic multifunctional monomers like N,N'-methylene bisacrylamide (MBA), 2-Propenoic acid, 2-methyl-, 1,1'-(1,10-decanediyl) ester (D3MA) or polyethyleneglycol diacrylates with two varying chainlengths (PEG-DA700, PEG-DA330). Furthermore several new multifunctional crosslinker with enhanced thermal and ageing stability have been synthesized and tested.

The advantage of several different building blocks with known characteristics is the possibility to tune the polymer to special needs of an application. For example, some polymer compositions have good conductivity at lower temperatures whereas other polymers develop better properties at elevated temperatures.
\end{abstract}

Keywords: fuel cell, proton exchange membrane, polymer electrolyte

\section{Introduction}

The proton exchange barrier or Proton Exchange Membrane (PEM) is the critical part of a fuel cell. The basic function of the membrane is to enable proton transport, while being simultaneously impermeable for electrons and gas. Typically, membranes for the PEM fuel cells (PEMFC) are made of perfluorocarbon-sulfonic acid monomers. The best known material of this class is Nafion which has a unique interpenetrating structure of hydrophobic perfluorocarbon regions providing thermal and chemical resistance, mechanical strength and diffusional resistance combined with hydrophilic regions of water clusters surrounding charged sulfonic acid groups which allow selective proton transport. For these reasons, Nafion is still considered the benchmark against which most of the new materials are compared [1].

At the molecular level, proton transport may follow two principal mechanisms: (a) diffusion mechanism via $\mathrm{H}_{3} \mathrm{O}^{+}$ion as a carrier and (b) proton hopping mechanism (Grotthuss transport) [2]. Contemporary PEMFCs are exclusively based on the vehicle mechanism. As a consequence, PEMFCs have inherent deficiencies, and a number of them are fundamental, originating from the membrane material.

Water content in the Nafion membrane must be kept within a narrow range. However, PEMFCs produce water as a by-product. Next, $\mathrm{H}^{+}$ions moving from the anode to the cathode pull water molecules by an electro-osmotic drag force. In addition, membrane suffers from evaporation of water at working temperatures of $60-90^{\circ} \mathrm{C}$. Nafion is unstable at temperatures over $100^{\circ} \mathrm{C}$, and effectively conducts protons only when imbibed by a sufficient amount of water, which limits the operating temperature of PEM fuel cells to around $80^{\circ} \mathrm{C}$. However an operating temperature above $100^{\circ} \mathrm{C}$ is a highly desirable goal. PEM membranes are not dimensionally stable since the material significantly swells upon water absorption.

The aim of this work is to create a membrane with a much lower water and fuel crossover than Nafion because than we can achieve higher conductivity with much thinner membranes even if the conductance is lower than Nafion. The idea is to membrane made of a rigid polymer to lower the permeability with perpendicular nano channels which are filled with a proton conducting polymer where conductivity is mainly achieved by the Grotthuss mechanism. In the first phase of our research different mono- and multifunctional monomers, which are commercially available and newly synthesized respectively, were screened for their properties in swelling, proton conductance and thermal stability.

\section{Experimental part}

\subsection{Materials}

Biaxially stretched polypropylene (PP) membranes from $3 \mathrm{M}$ with porosity of $80 \%$ were cut 
<smiles>C=CC(=O)NC(C)(C)CS(=O)(=O)O</smiles><smiles>C=CC(=C)C=CC(=C)C=CC(=C)CCCCC(=C)C=C</smiles><smiles>C=C(C)C(=C)N(CCCOCCCNC(=O)C(=C)C)CCC(C)(C)OCCNC(=O)C(=C)C</smiles>

Fig. 1. a) AMPS; b) Elastamine HE-1000; c) 4EG-DA $(n=4)$, PEG700-DA $(n \approx 13)$;

d) SR415 $(x+y+z=20)$; e) XL-1

into pieces of $20 \times 18 \mathrm{~mm}$. The membranes were impregnated with Triton X-100 when using water based formulations. Hydrophilic polyethersulfone (PES) membranes with porosity of $79 \%$ from PALL Life Sciences (Supor 200 Membrane, 0,2 $\mu \mathrm{m}$ pore size, $25 \mathrm{~mm}$ diameter) were used as received.

Methacrylic anhydride from Sigma-Aldrich was distilled before use. 2-acrylamido-2-methyl1-propanesulfonic acid (AMPS), tetra(ethylene glycol) diacrylate tech. (4EG-DA) and poly(ethylene glycol) diacrylate $\left(M_{n} 700\right)$ (PEG700-DA) was purchased from Sigma-Aldrich and used as received. Polyetheramine Elastamin HE-1000, sample received from Huntsman, ethoxylated(20) trimethylolpropane triacrylate (SR415), sample received from Sartomer, were used as received. (see Fig. 1) All other chemicals were from commercial suppliers and used as received.

\subsection{Synthesis of new crosslinker $X L-1$}

$8 \mathrm{~g}$ Elastamine $\mathrm{HE}-1000\left(\mathrm{M}_{\mathrm{w}} \approx 1000 \mathrm{~g} / \mathrm{mol}\right)$ was dissolved in $20 \mathrm{ml} \mathrm{CH} \mathrm{CH}_{2} \mathrm{Cl}_{2}$ and $3 \mathrm{~g}$ methacrylic anhydride in $20 \mathrm{ml} \mathrm{CH}_{2} \mathrm{Cl}_{2}$ was slowly added while keeping the temperature below $5^{\circ} \mathrm{C}$. Afterwards the solution was stirred for one hour at $5^{\circ} \mathrm{C}$ and one hour at room temperature. Methacrylic acid was removed by extraction with saturated $\mathrm{NaHCO}_{3}$ solution, 4methoxyphenol was added as stabilizer and the combined organic layers were evaporated to dryness.

\subsection{Membrane preparation}

The PP membranes were impregnated with Triton $\mathrm{X}-100$ by dipping them into a $1 \%$ solution of the surfactant in acetone.

2-Acrylamido-2-methylpropane sulfonic acid (AMPS) was mixed with different amounts of multifunctional monomer (crosslinker), Ciba Irgacure 2959 (photoinitiator) and water or NMP as solvent. The membranes were dip coated in the solution, plotted with paper to remove excessive surface wetness, put on a Teflon tray and covered with a Teflon foil. The samples were polymerized in an UViTron IntelliRay 600 UV flood curing system equipped with an UVB lamp useing $90 \%$ intensity for $30 \mathrm{~s}$ and afterwards extracted with water to remove unreacted monomer.

\subsection{Conductivity and swelling measurement}

Proton conductivity was measured in the plane of the nanomembrane samples using a 4-point method: direct current was measured between two gold plated inner electrodes placed $1 \mathrm{~cm}$ apart, while keeping the potential difference between two gold plated outer electrodes constant at $100 \mathrm{mV}$. Under these conditions no significant polarization of the electrodes is expected. Membrane samples, after the extraction of reaction residues for 24 hours in deionized water, were equilibrated in deionized water prior to the measurements. Ohmic resistance and membrane thickness measured by a contact gauge were converted into electrical conductivity, which should be 


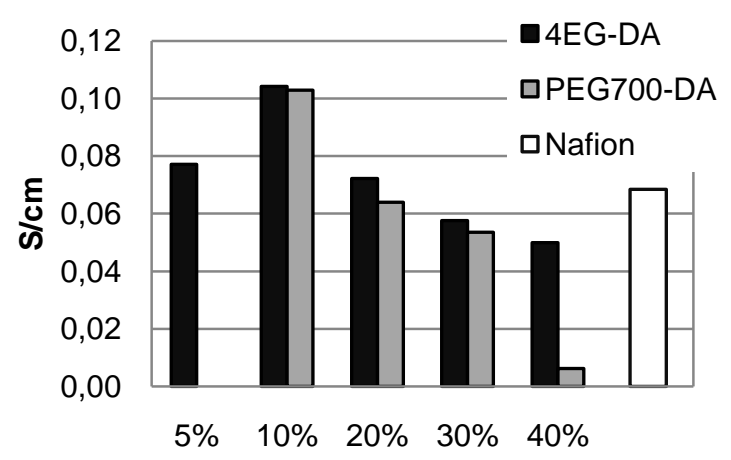

Fig. 2. Conductivity with difunctional crosslinker equal to the proton conductivity under the measuring conditions.

\section{Results and discussion}

Early experiments with AMPS and apolar crosslinker like decanediol dimethacrylate $\left(D_{3} M A\right)$ showed very poor results in terms of conductivity although the weight gain of the membranes was in a usual range compared to more polar crosslinker. The conductivity rapidly dropped to zero with increasing concentrations of apolar crosslinker which is an indication that the presence of water in the membrane is still necessary for good conductivity when using sulfonic acid based ionomers.

In several experiments we observed that higher monomer concentrations lead to higher weight gain and better conductivity. The PP membranes on the other hand were not strong enough to cope with the stress induced by the swelling when using water based AMPS solutions with 44 wt.\% and hydrophilic difunctional crosslinker like 4EG-DA or PEG700-DA. So we switched to PES membranes for these experiments. Fig. 2 shows the conductivity of different concentrations (mol\% in relation to AMPS) of crosslinker. In spite of the big difference in molecular weight both crosslinker offer similar performance and with a conductivity of $0.104 \mathrm{~S} / \mathrm{cm}$ for $10 \%$ of $4 E G-D A$ and $0.103 \mathrm{~S} / \mathrm{cm}$ for $10 \%$ of PEG700-DA both crosslinker could exceed the conductivity of Nafion with its $0.068 \mathrm{~S} / \mathrm{cm}$. However stability tests have shown that the PEG based diacrylates are thermally unstable. The acidic conditions inside the membrane might either accelerate the cleavage of the ether bonds of PEG or, which is more likely, the cleavage of the ester bond.

To reduce the amount of needed crosslinker in the proton conducting polymer network we tested two trifunctional hydrophilic crosslinker. SR415, a commercial crosslinker, is based on PEG with acrylate end groups like the two former crosslinker and $\mathrm{XL}-1$, which has to be synthesized, is based on PEG with methacrylamide end groups. We decided on using methacrylamides because amides are more stable in acidic environments than esters. Fig. 3 shows the conductivity of these two crosslinker in water based AMPS solutions (44 wt.\%) in PP membranes. The more polar crosslinker $\mathrm{XL}-1$ shows

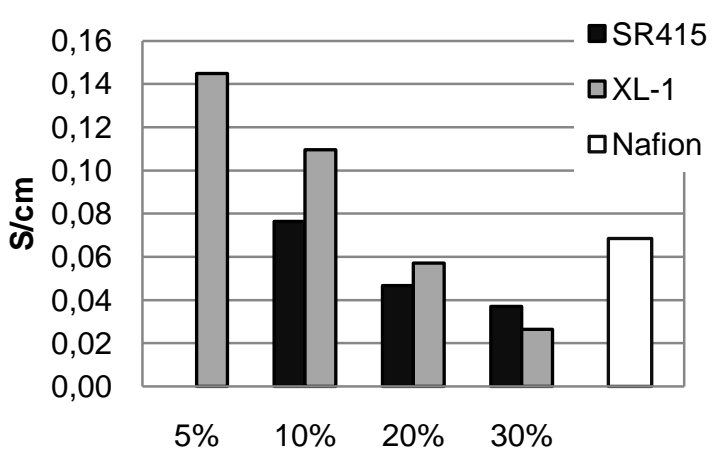

Fig. 3. Conductivity with trifunctional crosslinker

better performance than the slightly less polar SR415 although SR415 could outperform XL-1 at high concentrations. The highest conductivity in the tests is already achieved with $5 \% \mathrm{XL}-1$ which indicates that less crosslinker is needed when using multifunctional crosslinker. The conductivity of Nafion $(0.068 \mathrm{~S} / \mathrm{cm})$ could be more than doubled to $0.145 \mathrm{~S} / \mathrm{cm}$ for $5 \%$ of $\mathrm{XL}-1$.

\section{Conclusion and outlook}

We could by far exceed the conductivity of state of the art Nafion membranes with our crosslinked polymer networks. Especially newly synthesized multifunctional crosslinker performed very well in terms of conductivity. Hence we plan to syntesze several new multifunctional crosslinker with more than three functional groups. Especially poylethylenimines with different chain lengths and varying in the amount of conversion of the amino groups to (meth-) acrylamides are projected. Depending on the polymer substrate remaining amines may be reacted to link the conducting polymer to the substrate polymer.

The gained insights will be used to functionalize different inorganic materials and nanonporous membranes with proton conducting formulations to gain high proton conductivity and low gas and liquid permeability.

\section{Acknowledgements}

The research leading to these results has received funding from the European Community's FP7- NMP Programme, under the Project Acronym MultiPlat with Grant Agreement: N 228943 and the Austrian Federal Ministry of Science and Research. The authors would like to thank 3M for PP membrane samples and Huntsman and Sartomer for samples of crosslinker.

\section{References}

1. Hamrock, S.J. and M.A. Yandrasits, Proton Exchange Membranes for Fuel Cell Applications. 2006. 46(3): p. 219 - 244.

2. Hoogers, G., Membranes and lonomers, in Fuel Cell Technology Handbook G. Hoogers, Editor. 2002, CRC Press. p. 360 